\title{
Human brain organoids in Alzheimer's disease
}

\author{
You Jung Kang ${ }^{1}$, Hansang $\mathrm{Cho}^{2}$ \\ ${ }^{1}$ Department of Mechanical Engineering and Engineering Science, Department of Biological Sciences, and Center for Biomedical Engineering and \\ Science, University of North Carolina at Charlotte, Charlotte, NC, USA \\ ${ }^{2}$ Institute of Quantum Biophysics, Department of Biophysics, and Department of Intelligent Precision Healthcare Convergence, Sungkyunkwan \\ University, Suwon, Korea
}

Received: February 15, 2021

Revised: March 10, 2021

Accepted: March 15, 2021

Correspondence to:

Hansang Cho, Ph.D.

Institute of Quantum Biophysics,

Department of Biophysics, and

Department of Intelligent Precision

Healthcare Convergence,

Sungkyunkwan University, 2066

Seobu-ro, Jangan-gu, Suwon 16419,

Korea

E-mail: h.cho@g.skku.edu

\begin{abstract}
Alzheimer's disease (AD) is a progressive neurological disorder that typically involves neuronal damage leading to the deterioration of cognitive and essential body functions in aging brains. Major signatures of AD pathology include the deposition of amyloid plaques and neurofibrillary tangles, disruption of the blood-brain barrier, and induction of hyper-activated proinflammation in the brain, leading to synaptic impairment and neuronal loss. However, conventional pharmacotherapeutic modalities merely alleviate symptoms, but do not cure $A D$, partly because drug screening has used model systems with limited accuracy in terms of reflecting AD pathology in human brains. In this regard, several AD organoids have received substantial attention as alternatives to $A D$ animal models. In this review, we summarize the key characteristics required for the generation of a pathologically relevant $A D$ brain organoid. We also overview major experimental organoid models of AD brains, such as spheroids, three-dimensional (3D) bioprinted constructs, and 3D brain-on-chips, and discuss their strengths and weaknesses for AD research. This review will provide valuable information that will inspire future efforts to engineer authentic $A D$ organoids for the study of $A D$ pathology and for the discovery of novel $A D$ therapeutic strategies.
\end{abstract}

Keywords: Alzheimer disease; Brain organoid; Spheroid; Bioprinting; Microfluidics

\section{Introduction}

Alzheimer's disease $(\mathrm{AD})$, the most common cause of dementia, is a worldwide public health concern that occurs in a meaningful proportion of elderly individuals [1]. The total number of deaths caused by $\mathrm{AD}$ has steadily increased as current therapies merely rely on alleviating symptoms, not on curing $\mathrm{AD}[1]$. To develop a definitive cure for $\mathrm{AD}$, scientists have poured their efforts into the discovery of novel pathogenic mechanisms leading to neuronal loss and deterioration of cognitive function in aging brains [2]. Transgenic mice have been widely employed to study the implications of several genes associated with familial $\mathrm{AD}$ (FAD) for
$\mathrm{AD}$ progression [3]. For instance, transgenic mice with mutations in amyloid beta $(A \beta)$ precursor protein $(\mathrm{APP})$ and/or presenilin (PS) are gold-standard $\mathrm{AD}$ model systems as they recapitulate signature aspects of $\mathrm{AD}$, such as neuroinflammation and the deposition of $A \beta$ and hyperphosphorylated-Tau (pTau) in the brain [3-7]. Despite these similarities, a number of discrepancies compared to human $\mathrm{AD}$ brains have been reported [8]. Although the accumulation of $A \beta$ was observed, the formation of neurofibrillary tangles (NFTs) in neurons was not reproduced well at the same time in those animal models [8]. Moreover, the current transgenic mice models are only available to recapitulate $\mathrm{FAD}$, not sporadic $\mathrm{AD}$ ( $\mathrm{SAD}$ ), which accounts for approximately $97 \%$ of $\mathrm{AD}$

Copyright (C) 2021 The Organoid Society

This is an Open Access article distributed under the terms of the Creative Commons Attribution Non-Commercial License (http://creativecommons.org/licenses/bync/4.0/) which permits unrestricted non-commercial use, distribution, and reproduction in any medium, provided the original work is properly cited. 
cases [8]. To overcome the limitations of animal models having different genetic backgrounds from humans, humanized threedimensional (3D) $\mathrm{AD}$ models, also known as $\mathrm{AD}$ organoids, have been suggested as alternatives to animal models $[4,5,7,9-11]$.

$\mathrm{AD}$ organoids are $3 \mathrm{D}$ cell-cultured and self-organized $\mathrm{AD}$ models based on human cells or human induced pluripotent stem cells (iPSCs) expressing FAD or SAD-associated genes [4,5,7,912]. Various forms of $\mathrm{AD}$ organoids have been proposed that closely reproduce $\mathrm{AD}$ pathogenesis, including depositions of $\mathrm{A} \beta$ plaques and NFTs as well as promotion of neurodegenerative inflammation $[4,6,7]$. The organoids involve spheroids, 3D bioprinted brain tissues or vessels, brains-on-a-chip, and combined platforms $[4,5,7,9-11]$. The use of cell reprogramming systems, such as $\mathrm{AD}$ neurons derived from $\mathrm{AD}$ patient fibroblasts, allows reproduction of the genetic material of $\mathrm{AD}$ patients as well as $\mathrm{AD}$ pathology $[10,13]$. $\mathrm{AD}$ organoids enable dissection and reconstruction of extremely complex functions of healthy and/ or $\mathrm{AD}$ human brains that are otherwise not possible in animal or human models [4,5,7,9-11]. Furthermore, these miniaturized models can allow several robust and high-throughput analyses under a controllable microenvironment. The microarchitectures of $\mathrm{AD}$ organoids are relevant and simple, and they are capable of recapitulating specific biology, mechanisms, and development of neurological disorders. Overall, $\mathrm{AD}$ organoids represent excellent models for understanding the progression of both FAD and $\mathrm{SAD}$, which may contribute to the development of effective $\mathrm{AD}$ preventions and cures.

In this review, we explore details on $\mathrm{AD}$ organoids, in terms of (1) the major characteristics of $\mathrm{AD}$ to be implemented in $\mathrm{AD}$ organoids, (2) the design and preparation of various $\mathrm{AD}$ organoids, (3) advantages and potential challenges, and (4) future directions in this area.

\section{Major characteristics found in AD}

This section provides essential background regarding pivotal $\mathrm{AD}$ features to be replicated in $\mathrm{AD}$ models. The evaluation criteria are summarized in Table 1 [4-7,10,11,14-17].

\section{Accumulation of $A \beta$ aggregates}

The accumulation of insoluble $A \beta$ fibrils and plaques is the first checkpoint to determine the pathophysiological relevance of in vitro $\mathrm{AD}$ models [8]. The formation of insoluble amyloid fibrils and plaques from $\mathrm{A} \beta$ peptides has been considered as a primary characteristic found in $\mathrm{AD}$ patients at the early stages [18]. In normal conditions, APP is processed by $\gamma$-secretase, producing $\mathrm{P} 3$ fragments. In $\mathrm{AD}$ pathological conditions, APPs are truncated by $\beta$-secretase and $\gamma$-secretase, resulting in the production of various $A \beta$ peptides depending on the cleavage site by $\gamma$-secretase. Although $A \beta_{1-40}(\mathrm{~A} \beta 40)$ peptides are the most abundant form, comprising $80 \%-90 \%$ of $A \beta$ peptides, $A \beta_{1-42}$ (A $\beta 42$ ) peptides are neurotoxic as they are hydrophobic and prone to form aggregates, which further develop into oligomers, amyloid fibrils, and amyloid plaques, sequentially. In this regard, most in vitro $\mathrm{AD}$ models have utilized neuroprogenitor cells expressing FAD mutations, which are known to achieve higher $A \beta 42 / A \beta 40$ ratios and the formation of $\mathrm{A} \beta$ plaques [8]. The most popular $\mathrm{FAD}$ mutations are $\mathrm{APP}$ mutations (e.g., KM670/671NL [Swedish], V717I [London], and V717F [Indiana]) and/or PS mutations (e.g., PS1-M146L, PS1L166P, PS1-I213T, and PS2-N141I) [8]. In addition, SAD models with mutations in $\mathrm{AD}$ risk factors (e.g., $\mathrm{APOE}$ e4 or TREM2) also exhibited the robust formation of $A \beta$ plaques $[19,20]$. Therefore, $\mathrm{AD}$ models can be evaluated by validating the presence of an increased soluble $A \beta 42 / A \beta 40$ ratio and the accumulation of $A \beta$ oligomers, fibrils, and plaques in the models [18].

\section{Deposition of tau fibrils}

The formation of NFTs in neuronal cells is the second hallmark to be validated upon completion of $\mathrm{AD}$ model preparation [8]. Tau is a microtubule-binding protein that stabilizes microtubules in normal physiological conditions [8]. During $\mathrm{AD}$ progression, tau proteins are hyperphosphorylated, detached from microtubules, and formed into paired helical filaments (PHFs), followed by NFTs, which are frequently found in $\mathrm{AD}$ models and patient biopsies [21]. Several studies also revealed the formation of NFTs was highly correlated with axonal degeneration, mitochondrial dysfunction, and synaptic dysfunction, which further increase neurodegeneration [21]. Increased kinase activity (e.g., GSK-3 $\beta$ and CDK5) is known as a modulating mechanism to induce the phosphorylation on multiple sites of tau (more than 7-8 phosphates per tau), resulting in pTau $[22,23]$. Other modifications, such as proteolytic cleavage generating a S258-I360 fragment and O-GlcNac glycosylation in the S/T-P motif, also promote the formation of tau aggregations in $\mathrm{AD}$ brains [24,25]. Growing evidence shows that $A \beta$-driven increased glial activity produces proinflammatory factors that further increase tau accumulations in $\mathrm{AD}$ brains $[4,6]$. In addition, many $\mathrm{AD}$ patient brains exhibited aggregates of both $\mathrm{A} \beta$ and pTau, indicating the potential role of interactions between the two misfolded proteins in $\mathrm{AD}$. Given the strong correlation between pTau accumulation and $\mathrm{AD}$ pathology, increased levels of pTau and the presence of PHFs and NFTs are standard markers to evaluate in $\mathrm{AD}$ models [18]. 
Table 1. Major hallmarks to be validated in AD organoids

\begin{tabular}{|c|c|c|c|}
\hline Category & AD hallmarks & Evaluation methods & Validated models [Ref] \\
\hline \multirow[t]{6}{*}{$A \beta$ hypothesis } & $A \beta 42 / A \beta 40$ ratios $\uparrow$ & ELISA-A $\beta 40,42$ & AD iPSC spheroids [10] \\
\hline & & & APP/PS1 AD chips [4] \\
\hline & & & AD-BBB chips [7] \\
\hline & $A \beta$ oligomers/fibrils & ICC-A $\beta 42$ & APP/PS1 spheroids [11] \\
\hline & & WB-Aß42 & APP/PS1 AD chips [4] \\
\hline & $A \beta$ plaques & IHC-Aß42 & hA $\beta$ plaque mice [16] \\
\hline \multirow[t]{4}{*}{ Tauopathy } & pTau & ICC-pTau & AD iPSC spheroids [10] \\
\hline & & & APP/PS1 spheroids [11] \\
\hline & PHF/NFT-like & ICC-pTau & APP/PS1 3D AD [5] \\
\hline & & & APP/PS1 AD chips $[4,6]$ \\
\hline \multirow[t]{6}{*}{ Neuroinflammation } & Reactive astrocytes & IHC, ICC-GFAP & APP/PS1 mice [6] \\
\hline & & & APP/PS1 AD chips [6] \\
\hline & M1 microglia & ICC-CD11b, CD68 & APP/PS1 AD chips [4] \\
\hline & Proinflammatory chemokines & Multicytokine assay-CCL1, CCL2, CXCL1, CXCL10 & APP/PS1 AD chip [4] \\
\hline & Proinflammatory cytokines & Multicytokine assay-IL1 $\beta$, IL6, IL-8, TNF $\alpha$, IFN $\gamma$ & APP/PS1 AD chip [4] \\
\hline & & & AD-BBB chips [7] \\
\hline \multirow[t]{5}{*}{ Vascular dysfunction } & Tight junctions & ICC-ZO-1, $\beta$-catenin, VE-cadherin, claudin- 5 & BBB-spheroids [14] \\
\hline & & & AD-BBB chips [7] \\
\hline & & & BBB chips $[15,17]$ \\
\hline & Permeability $\uparrow$ & Translocation of dextran $\uparrow$ & AD-BBB chips [7] \\
\hline & & TEER $\downarrow$ & BBB chips [15] \\
\hline \multirow[t]{4}{*}{ Neurodegeneration } & Defect in neuronal functions & Intracellular $\mathrm{Ca}^{2+}$ level & APP/PS1 AD chips [4] \\
\hline & Neuronal population $\downarrow$ & ICC-Tuj1, MAP2 & APP/PS1 AD chips [4] \\
\hline & Viability $\downarrow$ & LDH assay & APP/PS1 AD chips $[4,6]$ \\
\hline & & EthD-1 staining & AD-BBB chips [7] \\
\hline
\end{tabular}

$A D$, Alzheimer's disease; $A \beta 42, A \beta_{1-42} ; A \beta 40, A \beta_{1-40} ; E L I S A$, enzyme-linked immunosorbent assay; iPSC, induced pluripotent stem cell; $A P P$, $A \beta$ precursor protein; PS, presenilin; BBB, blood-brain barrier; ICC, immunocytochemistry; WB, western blotting; IHC, immunohistochemistry; pTau, hyperphosphorylated-Tau; PHF, paired helical filament; NFT, neurofibrillary tangle; IL, interleukin; TNF, tumor necrosis factor; IFN, interferon; TEER, transendothelial electrical resistance measurement; LDH assay, lactate dehydrogenase activity assay.

\section{Neuroinflammation}

The prominent activation of innate immune cells can be a promising marker to be monitored, as their phenotypes are closely correlated with the severity of $\mathrm{AD}$ [26]. Among the innate immune cells, astrocytes and microglia are two major components involved in the inflammatory responses to $\mathrm{AD}$ ques $[4,12]$. In the early stages of $A D$ where protein $A \beta$ and tau aggregates are starting to be formed in the brain, the polarization of anti-inflammatory astrocytes and microglia appears to be dominant. Astrocytes serve neuroprotective roles by secreting neurotransmitters supporting neuronal activity and removing toxins around neuronal cells [27]. Microglia also play neuroprotective roles by phagocytosing damaged synaptic parts, called synaptic pruning [12,28]. However, chronic exposure to $\mathrm{AD}$ conditions can alter the phenotype of both microglia and astrocytes, promoting proinflammation and neurodegeneration in $\mathrm{AD}$ brains [4]. In the later stages of $\mathrm{AD}$, a number of studies demonstrated the induction of hyper-reactive astrocytes, promoting the significant levels of oxidative stress (hydrogen peroxide $\left[\mathrm{H}_{2} \mathrm{O}_{2}\right]$ and nitric oxide [NO]) and the production of proinflammatory cytokines (interleukin $[\mathrm{IL}]-1 \beta$, IL6, tumor necrosis factor $[\mathrm{TNF}]-\alpha$, interferon $[\mathrm{IFN}] \gamma$, etc.) and chemokines (CCL1, CCL2, CXCL1, etc.) that further activate other proinflammatory components of the innate immune system $[4,6]$. Moreover, microglia infiltrate into regions with $A \beta$ and NFT accumulations and display a detrimental phenotype that exacerbates $\mathrm{AD}$ pathology by increasing oxidative stress (superoxide $\left[\mathrm{O}_{2}\right], \mathrm{NO}$ ), tau aggregates, and proinflammatory mediator levels (IL-1 $\beta, \mathrm{IL}-6, \mathrm{TNF}-\alpha$, etc.) [4]. In this regard, AD models can be evaluated by investigating the phenotype of innate immunity and assessing the increased soluble factors from innate immune cells.

\section{Neurovascular dysfunction}

Neurovascular dysfunction is a distinctive feature frequently 
observed in $\mathrm{AD}$ patient brains, which show an increased influx of neurotoxic blood-derived debris, microbial pathogens, and proinflammatory responses, all of which exacerbate neurodegeneration [29]. Neurovascular abnormalities have been strongly correlated with blood-brain barrier (BBB) disruption and attributed to genetic $\mathrm{AD}$ risk factors, $\mathrm{A} \beta /$ tau pathology, and the degeneration of $\mathrm{BBB}$ components, such as endothelial cells and pericytes [29]. According to recent positron emission tomography and magnetic resonance imaging studies, $\mathrm{BBB}$ disruption was observed in approximately $40 \%$ to $80 \%$ of $\mathrm{AD}$ patients, particularly in the hippocampus region, a center for memory and learning processes $[29,30]$. Several studies have shown that $\mathrm{AD}$ patients possessing an $\mathrm{AD}$ risk factor such as APOE e4 have higher levels of cyclophilin $A$ and matrix metalloproteinase 9 (MMP9) in the endothelium and pericytes of $\mathrm{BBB}[31,32]$. Follow-up studies showed that the activation of cyclophilin A-MMP9 axis decreased BBB integrity $[32,33]$. Amyloid and tau depositions on the vascular wall can also decrease $\mathrm{BBB}$ integrity by inducing endothelial degeneration $[34,35]$. In addition, reduced pericyte numbers around the $\mathrm{BBB}$ and increased infiltrated-macrophages can increase $\mathrm{BBB}$ permeability $[33,36]$. BBB disruption can be confirmed by detecting any decrease in tight-junction markers (e.g., ZO$1, \beta$-catenin, VE-cadherin, and claudin-5) [14]. The direct consequences of a leaky BBB can be evaluated by monitoring any increase in the permeability coefficient of fluorescent probes or a decrease in transendothelial electrical resistance across the $\mathrm{BBB}$ $[7,15]$. In addition, the consequences of $\mathrm{BBB}$ disruption can be markers of neurovascular dysfunction in $\mathrm{AD}$; examples include any increased proinflammatory mediators, accumulated bloodderived proteins (e.g., albumin, plasminogen, fibrinogen, and immunoglobulin $\mathrm{G}$ ), and deposited neurotoxic debris or microbial pathogens [29].

\section{Neurodegeneration}

A significant reduction in brain mass is a major hallmark of latestage $\mathrm{AD}$, leading to clinical symptoms such as progressive decline in memory, thinking, language, and essential body functions [1]. Since the assessment of clinical symptoms is impossible within in vitro models, the detection of neuronal damage and neuronal loss is an alternative criterion to be validated in $\mathrm{AD}$ models. The induction of synaptic impairment followed by the loss of neural function has been observed in both $\mathrm{AD}$ patients and in vitro $\mathrm{AD}$ models $[4,37,38]$. Evidence of synaptic impairment involves reductions in major synaptic proteins (e.g., calsyntenin, GluR, neurexin, syntaxin, and synapsin) that participate in core synaptic processes such as dendritic spine assembly, postsynaptic $\mathrm{Ca}^{2+}$ signaling, synaptic transmission, synaptogenesis, and presynaptic differentiation [39]. Decreased neural activity can be assessed by the neural responses toward optical, electrical, or chemical stimulation. For instance, several studies showed reduced intracellular $\mathrm{Ca}^{2+}$ levels in $\mathrm{AD}$ neural cells in response to an action potential buffer [4]. The most apparent evidence of neurodegeneration is the reduction of neural population in $\mathrm{AD}$ models. To this end, a number of studies validated neurodegeneration by showing a decrease in neuronal markers or the viability of $\mathrm{AD}$ models $[4,6,7]$.

\section{AD spheroids}

Multicellular spheroids represent a simple form of organoids used to predict pharmacokinetics and the therapeutic efficacy of candidate drugs in the brain $[10,11,14]$. A spherical cluster of neural cells retains a 3D configuration and closely mimics the functional and structural properties of the brain tissue $[10,11]$. In addition, BBB-spheroids can be prepared by wrapping the brain spheroid with an additional layer of brain endothelial cells that recapitulate the penetration of medicines or pathogens across the BBB [14]. Therefore, spheroids serve as an excellent brain disease model for the preclinical evaluation of therapeutics targeting neurological disorders, including $\mathrm{AD}$.

A method to create $\mathrm{AD}$ spheroids is to culture $\mathrm{AD}$ neuronal cells on non-cell adherent surfaces or hydrogels not containing integrin-binding motifs (e.g., agarose, polyethylene glycol, or F127DA hydrogels), which facilitate cell-cell interactions leading to the formation of cell aggregates [40-42]. Lee et al. [10] cultured $\mathrm{AD}$ patient-derived neural cells on ultra-low-attachment plastic plates and generated 3D human $\mathrm{AD}$ neurospheroids. The resultant $\mathrm{AD}$ patient neurospheroids displayed $\mathrm{AD}$ signatures such as the production of $A \beta 40$ and $A \beta 42$ and deposition of pTau. These personalized $\mathrm{AD}$ neurospheroids in an array format were used to screen various $\mathrm{AD}$ treatments including $\mathrm{BACE} 1$ and $\gamma$-secretase inhibitors in a high-throughput manner. However, the neurospheroids prepared by this method were not homogeneous in size and shape. To precisely control the size and shape of spheroids to achieve more consistent results, a number of studies employed a hanging drop or a micro-mold method [40,42]. In the hanging drop method, cells are in the culture medium suspended to the underside of petri dish lids [43]. Under the force of gravity, cells are congregated onto the bottom part of the hanging drop and form spheroids. The size of spheroids can be adjusted by changing the cell seeding density. Nzou et al. [14] showed a human cortex spheroid mimicking a functional $\mathrm{BBB}$ prepared by hanging drop culture. The resultant BBB spheroid was employed to test adverse effects of inorganic mercury on the human cortex 
region. The micro-mold method can also be used to determine the size and shape of spheroids by adjusting cell culture mold design $[40,42]$. Jorfi et al. [11] cultured human neural progenitor cells (ReN) overexpressing GFP (control) or APP/PS1-GFP (AD) in uniformly sized polydimethylsiloxane (PDMS) microwells (Fig. 1A). This study achieved notable neurite outgrowth from the spheroids. In addition, $\mathrm{AD}$ spheroids exhibited higher expression levels of $A \beta 42$ and pTau than control spheroids.

Taken together, $\mathrm{AD}$ spheroids are advantageous as the preparation methods are simple and readily accessible for most labs, enabling efficient mass production. The major disadvantage of spheroids is the lack of diffusion of nutrients and oxygens in the core if their size exceeds $500 \mu \mathrm{m}$, leading to the formation of a necrotic core [44]. To solve this limitation, recent studies introduced perfusable vascular units into the spheroids, which supplied nutrients and oxygen to the deeper parts of spheroids [45].

\section{D-bioprinted AD brains}

The human brain is the most complex organ in the body over a thousand sub-regions, serving distinctive functions. Multiple components relevant for remodeling human brains, such as the cell population, density, orientation, and microenvironments, vary depending on the region. In the context of complexity, $3 \mathrm{D}$ bioprinting is a promising technique to construct $\mathrm{AD}$ brain models, particularly those associated with $\mathrm{BBB}$, as it allows the layer-by-layer deposition of cell-laden bioink with different compositions [9].

Inkjet bioprinting is a technique to pattern multiple droplets of low-viscous and soft bioink on the substrate [46]. Droplet formation can be controlled by a piezoelectric or electrostatic actuator, which applies impulsive voltage causing a pressure pulse on the nozzle orifice. The instantaneous volume increase of bioink caused by the pressure pulse results in the ejection of bioink from the nozzle and its deposition on the substrate. For instance, Lorber et al. [47] successfully printed adult rat ganglia and glial cells using a piezoelectric inkjet printer. Thermal actuators are another system used to control the formation of cell-laden droplets on the substrate by creating heat pulses, inducing partial vaporization, and generating pressure pulses for the ejection of bioink. Xu et al. [48] employed thermal inkjet printing to create a 3D neural sheet composed of primary neural cells from rat hippocampal and cortical tissues and fibrin gels. However, several studies reported that the frequencies $(15-25 \mathrm{kHz})$ of electric pulses and the local temperatures $\left(-300^{\circ} \mathrm{C}\right)$ of heat pulses could reduce the viability of mammalian cells during printing, which may limit the application of inkjet printing to create functional 3D brain organoids. Recently,
Sharma et al. [49] patterned 3D neural tissue with neuroprogenitor cells derived from iPSCs on the substrate under mild conditions using a microfluidic-based inkjet platform. Instead of using thermal or piezoelectric control, this system applied specific pressures to each channel to control the crosslinking and printing rate. Cell-laden bioink (a mixture of fibrinogen, alginate, and genipin) was mixed with a crosslinker at the junction of bioink and crosslinker channels to initiate gel solidification at the printhead of microfluidics. The cell-laden hydrogels were then transferred onto the substrate and formed into an array of semi-spherical neural tissue. Inkjet bioprinting allows high-resolution cell placement in the pico-liter range; however, the use of low-viscosity bioink limits the ability to pile up multiple layers of bioink in the Z-direction.

Extrusion-based bioprinting is a 3D bioprinting technique with viscous bioink that deposits a continuous filament and builds multiple layers. Gu et al. [50] generated a cubic-shaped neural mini-tissue by microextrusion bioprinting of human neural stem cells laden with bioink including the polysaccharides alginate, agarose, and carboxymethyl chitosan. The stiffness of the resultant mini-tissue (0.8-7.5 kPa) was comparable to that of human brains, as well as in vivo models. In addition, the printed neural stem cells were differentiated into GABAergic neurons, astrocytes, and oligodendrocytes. Miller et al. [51] printed a 3D filament of carbohydrate glass surrounded by a hydrogel encapsulating mouse embryonic cells. The filament is a sacrificial template creating a lumen area so that human umbilical vein endothelial cells were added and formed into vascular structures surrounded by tissue. Furthermore, the use of a coaxial nozzle composed of an outer and inner needle can extrude a two-layered filament with the inner layer to be removed later so that enable to create vascular structures in a single step [52]. Gao et al. [53] utilized coaxial extrusion to generate a blood vessel structure with endothelial progenitor cells (EPCs) covered by the vascular-tissue derived decellularized extracellular matrix (VdECM) (Fig. 1B). The hybrid bioink with EPCs and VdECM was loaded into the shell nozzle while Pluronic F-127 was in the core nozzle. Upon the completion of extrusion, the outer layer was solidified by thermal gelation and the inner layer was chemically removed by $\mathrm{CaCl} 2$ solution.

In this study, the resultant blood vessel along with a proangiogenic drug (atorvastatin) was successfully implemented in the ischemic limbs of mice and contributed to limb salvage. The major drawback of extrusion is the low speed of building up 3D structures and its limitation to viscous bioink, unlike other bioprinting methods; however, extrusion would be advantageous for constructing continuous multiple-layered vascular structures in a single step [9]. With improvements of the mechanical properties of hydrogel bioink, bioprinted models could be further 
employed not only for in vitro drug screening, but also for vascular replacements [53].

Overall, the 3D bioprinting technique can generate personalized 3D neural tissues or BBB constructs by stacking either cell-laden building blocks or extruded filaments on the substrate. It should be noted that there is no standard $\mathrm{AD}$ model prepared by $3 \mathrm{D}$ bioprinting as the current bioprinting technique is still in the early stages of development. Nonetheless, there is tremendous potential for improvement to create novel $3 \mathrm{D}$ models of $\mathrm{AD}$ brain tissues by $3 \mathrm{D}$ bioprinting in the future.

\section{AD brains-on-chips}

Neural cell culture in microfluidic devices, also referred as brainson-chips, have received extensive attention for their capability to miniaturize human brains. In this platform, multiple cells can be cultured within district compartments connected through networks of channels $[4,7,17,54]$. Therefore, this organoid enables the compartmentalization of each brain region, thereby simplifying the extremely complex functions of healthy and/or diseased human brains. Since the miniaturized devices are very thin and optically transparent, other optical, optochemical, and electrical measurement techniques have been integrated in microfluidics. Therefore, chip-based AD models allow multiple robust and highthroughput analyses in parallel.

Microfluidics has emerged as a single-cell culture platform that closely mimics the key cellular activities of specific cell types contributing to $\mathrm{AD}$ pathology $[4,7]$. In addition, microfluidicbased cell culture is advantageous in terms of the liquid handling system, which enables not only continuous supply/removal of nutrients and waste, but also the generation of stable chemical gradients for single-cell chemotaxis. Cho et al. [54] developed a chemotactic microfluidic platform that allowed the separation of stimulated human microglia subpopulations in vitro according to the use of soluble $A \beta$ or insoluble $A \beta$, extracted from $A D$ brain tissues. In addition, they further created an in vitro $3 \mathrm{D}$ cylindrical capillary-like structured BBB integrated with chemotactic channels, allowing a robust screening of BBB-targeting drugs targeting neurological disorders including $\mathrm{AD}$ [17]. This singlecultured microfluidic platform enabled the dissection and reconstruction of specific cellular responses toward $\mathrm{AD}$ que; however, the single-culture models did not consider multicellular crosstalk in $\mathrm{AD}$ pathology.

The advances in cell culture within microfluidics enable the construction of multicellular systems and increase the feasibility of $\mathrm{AD}$ models. Park et al. [4] engineered a 3D human $\mathrm{AD}$ brain model along with innate immunity by co-culturing human neuroprogenitor cells expressing APP/PS1 and adult microglial cells in a 3D microfluidic platform (Fig. 1C). In this model, two compartments for APP/PS1 neuroprogenitor cells and microglia were linked by multiple channels, allowing microglial migration in response to soluble factors from the $\mathrm{AD}$ chamber with neuroprogenitor cells. This $\mathrm{AD}$ model provided key representative $\mathrm{AD}$ features: pathological accumulation of $\mathrm{A} \beta$ and pTau, and microglial proinflammation leading to damage of $\mathrm{AD}$ neurons. This model was further evolved with the use of iPSCmicroglia to explore the pivotal roles of TREM2 in the migration and phagocytic activities in response to soluble $A \beta, A \beta$ plaques, and $3 \mathrm{D}$ cultured $\mathrm{AD}$ brain models. Shin et al. [7] combined a $3 \mathrm{D}$ $\mathrm{AD}$ model with $\mathrm{APP} / \mathrm{PS} 1$ neuroprogenitor cells and a 3D tubular $\mathrm{BBB}$ with hCEMC/D3 in a microfluidics platform to explore the impact of $\mathrm{BBB}$ dysfunction in $\mathrm{AD}$ pathology (Fig. 1D). Two parallel channels (an $\mathrm{AD}$ compartment and a $\mathrm{BBB}$ compartment) were connected by additional chambers with collagens allowing crosstalk between cells in the $\mathrm{AD}$ and $\mathrm{BBB}$ compartments. This study revealed that the increased production of $A \beta$, MMP2, and IFN $\gamma$ in the $3 \mathrm{D} A D$ models contributed to the induction of reactive oxygen species and reduction of tight junctions in 3D BBB. This in vitro model validated the major signature of $A D$, including an increase in $\mathrm{BBB}$ permeability leading to an increased influx of neurotoxins through the impaired BBB, followed by neuronal damage. In this regard, multicellular $\mathrm{AD}$ models in microfluidics enabled the integration of multiple functions in a single platform, which significantly improved both the physiological relevance and the efficacy of analyses in comparison to other in vitro models.

Taken together, the use of microfluidics allows the precise modulation and simplification of intricate biological parameters in the study of $\mathrm{AD}$ brains [4,5,7,9-11,55]. In addition, microfluidicbased $\mathrm{AD}$ models have been combined with other analytic tools to provide tractable and quantifiable platforms for the study of $\mathrm{AD}$ pathology in both qualitative and quantitative manners. Therefore, chip-based $\mathrm{AD}$ models represent excellent platforms for the study of extremely complex functions of $\mathrm{AD}$ human brains that were otherwise not possible in animal or human models. A major challenge of cell culture in microfluidics is the use of PDMS, which absorbs significant amount of nutrients and testing molecules, leading to dilution of components in the culture medium [56]. Another concern is the maintenance of physiologically relevant $\mathrm{pH}$ and oxygen levels inside microfluidics due to the use of minute volumes of media for the cell culture [56]. To this end, the culture medium should be replaced routinely, which can be automated by a perfusion system [57]. Even if the preparation of microfluidics requires complex and specialized equipment that is not usually affordable for most cell culture laboratories, enormous efforts 

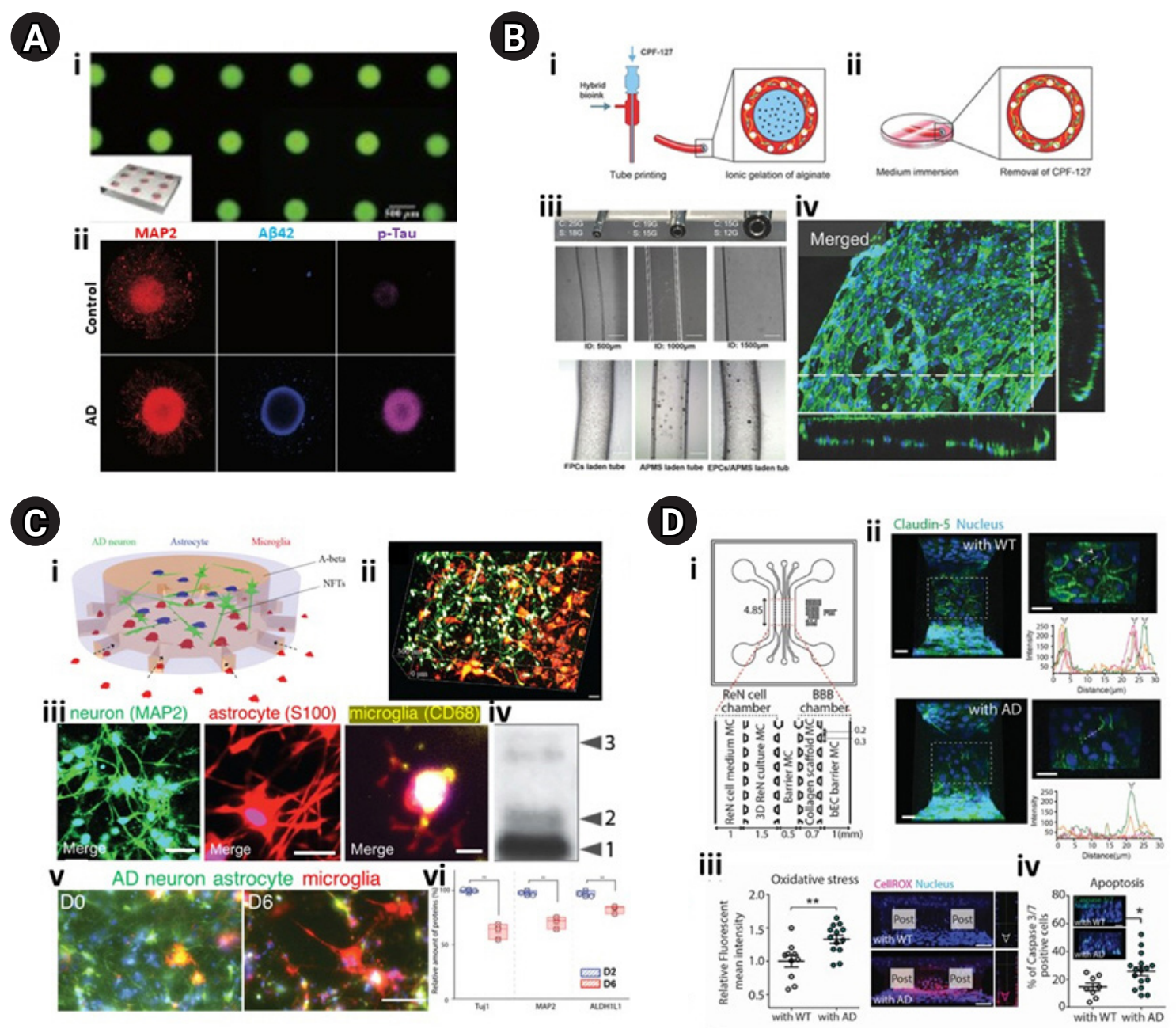

Fig. 1. Summary of major human brain organoids for the study of Alzheimer's disease (AD). (A) AD neurospheroids. (i) $A D$ neurospheroids were prepared in an array of polydimethylsiloxane microwells. (ii) Validation of $A D$ signatures, such as accumulations of $A \beta_{1-42}(A \beta 42)$ and hyperphosphorylated-Tau (pTau) in AD spheroids. Modified from Jorfi et al. [11]. (B) Bioprinted three-dimensional (3D) brain vascular structures. (i, ii) Schematic illustrations of coaxial extrusion to create a blood vascular structure. (iii) Conditions of extrusion to control size of vascular structure encapsulating endothelial progenitor cells. (iv) Fluorescent images representing intercellular junctions (CD31, green) and nucleus (4'6-diamidino-2-phenylindole [DAPI], blue) to confirm the integrity of blood vessels. Modified from Gao et al. [53]. (C) $3 D$ AD brain with innate immunity-on chips. (i) Schematic illustration of the AD chip configuration with $3 D$ AD brain (central chamber) connected to microglia region (annular chamber). (ii) Fluorescent images showing the migration of microglia (red) to AD brain region (green). (iii) Fluorescent images representing the presence of neurons (microtubule-associated protein 2 [MAP2], green), astrocytes (S100, red), and infiltrated microglia (CD68, yellow) in the AD brain model. (iv) Western blotting data confirming the expression of $A \beta$ aggregates in the AD brain model. ( $v$, vi) Neurodegeneration by microglia in 6 days. Modified from Park et al. [4] with permission from Springer Nature. (D) 3D AD brain with blood-brain barrier (BBB)-on chips. (i) Schematic representation of the AD chip design with 3D AD brain (left side) connected to 3D BBB region (right side). (ii) Fluorescent images showing the reduction of tight junction (Claudin-5) in $A D$ models. Validation of damaged $B B B$ in $A D$ by showing (iii) the increased reactive oxygen species and (iv) apoptotic population in $A D$ models. Modified from Shin et al. [7].

have been invested in achieving lower-cost and more standardized systems for microfluidic-based 3D cell culture.

\section{Outlook}

$\mathrm{AD}$ is a progressive and irreversible brain disease threatening the lives of millions of people throughout the world [1]. However, most recently-developed treatments for $\mathrm{AD}$ have failed to show any therapeutic efficacy in clinical phases even if they achieved impressive outcomes in preclinical trials with animal models [2]. In this review, we highlighted major human brain organoids for the study of $\mathrm{AD}$ pathology and the discovery of promising 
therapeutics for $\mathrm{AD}$. Recently, human $\mathrm{AD}$ brain organoids have emerged as alternatives as promising preclinical models since they can precisely recapitulate the architecture and functionality of human $\mathrm{AD}$ brains [4,5,7,9-11]. In addition, brain organoids provide tractable cellular platforms in combination with other analytical techniques that enable robust and accurate screening of drug candidates for $\mathrm{AD}$ in a controllable manner. The utilization of $\mathrm{AD}$ patient-driven iPSCs further improves physio-pathological features, both for FAD and SAD $[10,13]$. Brain organoids have recently been combined with other organs to form body organoids for testing the systemic effects of drug candidates [58,59]. Given the rapid progress of the development of $\mathrm{AD}$ organoids, we believe that they will offer novel experimental platforms to understand the nature of brain involvement in $\mathrm{AD}$ pathology and to accelerate the discovery of novel cures for $\mathrm{AD}$ in the near future.

\section{Notes}

\section{Conflict of interest}

No potential conflict of interest relevant to this article was reported.

\section{Funding}

This research was funded by NRF-2020R1A2C2010285, NRF2018M3C7A1056896, MOTIE-20012352 (the Ministry of Trade, Industry \& Energy, the Technology Innovation Program) (HC), and NIH AG059236-01A1 (YJK).

\section{Author contributions}

Conceptualization: HC, Funding acquisition: HC, Software: YJK, Supervision: HC, Visualization: YJK, Writing-original draft: all authors, Writing-review \& editing: all authors.

\section{Additional contributions}

We thanks Charles Lee (UNC Charlotte) for supporting our project.

\section{ORCID}

You Jung Kang, https: / / orcid.org/0000-0002-6847-3002

Hansang Cho, https://orcid.org/0000-0003-1829-2462

\section{References}

1. Prince M, Wimo A, Guerchet M, Ali GC, Wu YT, Prina M. World Alzheimer Report 2015: the global impact of dementia. An analysis of prevalence, incidence, cost and trends. London: Alzheimer's Disease International; 2015.

2. Kang YJ, Diep YN, Tran M, Cho H. Therapeutic targeting strategies for early- to late-staged Alzheimer's disease. Int J Mol Sci 2020;21:9591.

3. Elder GA, Gama Sosa MA, De Gasperi R. Transgenic mouse models of Alzheimer's disease. Mt Sinai J Med 2010;77:69-81.

4. Park J, Wetzel I, Marriott I, Dreau D, D’Avanzo C, Kim DY, et al. A 3D human triculture system modeling neurodegeneration and neuroinflammation in Alzheimer's disease. Nat Neurosci 2018;21:941-51.

5. Kim YH, Choi SH, D’Avanzo C, Hebisch M, Sliwinski C, Bylykbashi E, et al. A 3D human neural cell culture system for modeling Alzheimer's disease. Nat Protoc 2015;10:985-1006.

6. Chun H, Im H, Kang YJ, Kim Y, Shin JH, Won W, et al. Severe reactive astrocytes precipitate pathological hallmarks of Alzheimer's disease via $\mathrm{H} 2 \mathrm{O} 2$-production. Nat Neurosci 2020;23:1555-66.

7. Shin Y, Choi SH, Kim E, Bylykbashi E, Kim JA, Chung S, et al. Blood-brain barrier dysfunction in a $3 \mathrm{D}$ in vitro model of Alzheimer's disease. Adv Sci (Weinh) 2019;6:1900962.

8. Drummond E, Wisniewski T. Alzheimer's disease: experimental models and reality. Acta Neuropathol 2017;133:155-75.

9. Antill-O’Brien N, Bourke J, O'Connell CD. Layer-by-layer: the case for 3D bioprinting neurons to create patient-specific epilepsy models. Materials (Basel) 2019;12:3218.

10. Lee HK, Velazquez Sanchez C, Chen M, Morin PJ, Wells JM, Hanlon EB, et al. Three dimensional human neuro-spheroid model of Alzheimer's disease based on differentiated induced pluripotent stem cells. PLoS One 2016;11:e0163072.

11. Jorfi M, D’Avanzo C, Tanzi RE, Kim DY, Irimia D. Human neurospheroid arrays for in vitro studies of Alzheimer's disease. Sci Rep 2018;8:2450.

12. McQuade A, Kang YJ, Hasselmann J, Jairaman A, Sotelo A, Coburn M, et al. Gene expression and functional deficits underlie TREM2-knockout microglia responses in human models of Alzheimer's disease. Nat Commun 2020;11:5370.

13. Ochalek A, Mihalik B, Avci HX, Chandrasekaran A, Teglasi A, Bock I, et al. Neurons derived from sporadic Alzheimer's disease iPSCs reveal elevated TAU hyperphosphorylation, increased amyloid levels, and GSK3B activation. Alzheimers Res Ther 2017;9:90.

14. Nzou G, Wicks RT, Wicks EE, Seale SA, Sane CH, Chen A, et al. Human cortex spheroid with a functional blood brain barrier for high-throughput neurotoxicity screening and disease modeling. Sci Rep 2018;8:7413.

15. Park TE, Mustafaoglu N, Herland A, Hasselkus R, Mannix R, FitzGerald EA, et al. Hypoxia-enhanced Blood-Brain Barrier Chip recapitulates human barrier function and shuttling of drugs and antibodies. Nat Commun 2019;10:2621. 
16. Pedrero-Prieto CM, Flores-Cuadrado A, Saiz-Sanchez D, Ubeda-Banon I, Frontinan-Rubio J, Alcain FJ, et al. Human amyloid- $\beta$ enriched extracts: evaluation of in vitro and in vivo internalization and molecular characterization. Alzheimers Res Ther 2019;11:56.

17. Cho H, Seo JH, Wong KH, Terasaki Y, Park J, Bong K, et al. Three-dimensional blood-brain barrier model for in vitro studies of neurovascular pathology. Sci Rep 2015;5:15222.

18. Kametani F, Hasegawa M. Reconsideration of amyloid hypothesis and tau hypothesis in Alzheimer's disease. Front Neurosci 2018; 12:25.

19. Ulrich JD, Holtzman DM. TREM2 function in Alzheimer's disease and neurodegeneration. ACS Chem Neurosci 2016; 7:420-7.

20. Huang Y, Mahley RW. Apolipoprotein E: structure and function in lipid metabolism, neurobiology, and Alzheimer's diseases. Neurobiol Dis 2014;72 Pt A:3-12.

21. Imahori K, Uchida T. Physiology and pathology of tau protein kinases in relation to Alzheimer's disease. J Biochem 1997;121:179-88.

22. AugustinackJC, Sanders JL, Tsai LH, Hyman BT. Colocalization and fluorescence resonance energy transfer between cdk5 and AT8 suggests a close association in pre-neurofibrillary tangles and neurofibrillary tangles. J Neuropathol Exp Neurol 2002;61:557-64.

23. Sengupta A, Novak M, Grundke-Iqbal I, Iqbal K. Regulation of phosphorylation of tau by cyclin-dependent kinase 5 and glycogen synthase kinase-3 at substrate level. FEBS Lett 2006;580:5925-33.

24. Li X, Lu F, Wang JZ, Gong CX. Concurrent alterations of O-GlcNAcylation and phosphorylation of tau in mouse brains during fasting. Eur J Neurosci 2006;23:2078-86.

25. Wang Y, Martinez-Vicente M, Krüger U, Kaushik S, Wong E, Mandelkow EM, et al. Tau fragmentation, aggregation and clearance: the dual role of lysosomal processing. Hum Mol Genet 2009; 18:4153-70.

26. Kinney JW, Bemiller SM, Murtishaw AS, Leisgang AM, Salazar AM, Lamb BT. Inflammation as a central mechanism in Alzheimer's disease. Alzheimers Dement (N Y) 2018;4:57590.

27. Fakhoury M. Microglia and astrocytes in Alzheimer's disease: implications for therapy. Curr Neuropharmacol 2018;16:50818.

28. Keren-Shaul H, Spinrad A, Weiner A, Matcovitch-Natan O, Dvir-Szternfeld R, Ulland TK, et al. A unique microglia type associated with restricting development of Alzheimer's disease. Cell 2017;169:1276-90.e17.
29. Sweeney MD, Sagare AP, Zlokovic BV. Blood-brain barrier breakdown in Alzheimer disease and other neurodegenerative disorders. Nat Rev Neurol 2018;14:133-50.

30. Brundel M, Heringa SM, de Bresser J, Koek HL, Zwanenburg JJ, Jaap Kappelle L, et al. High prevalence of cerebral microbleeds at 7Tesla MRI in patients with early Alzheimer's disease. J Alzheimers Dis 2012;31:259-63.

31. Wood H. APOE* $\varepsilon 4$ is linked to BBB breakdown. Nat Rev Neurol 2020;16:350.

32. Bell RD, Winkler EA, Singh I, Sagare AP, Deane R, Wu Z, et al. Apolipoprotein E controls cerebrovascular integrity via cyclophilin A. Nature 2012;485:512-6.

33. Halliday MR, Rege SV, Ma Q, Zhao Z, Miller CA, Winkler EA, et al. Accelerated pericyte degeneration and blood-brain barrier breakdown in apolipoprotein E4 carriers with Alzheimer's disease. J Cereb Blood Flow Metab 2016;36:216-27.

34. Hartz AM, Bauer B, Soldner EL, Wolf A, Boy S, Backhaus $\mathrm{R}$, et al. Amyloid- $\beta$ contributes to blood-brain barrier leakage in transgenic human amyloid precursor protein mice and in humans with cerebral amyloid angiopathy. Stroke 2012;43:514-23.

35. Zenaro E, Piacentino G, Constantin G. The blood-brain barrier in Alzheimer's disease. Neurobiol Dis 2017;107:41-56.

36. Sengillo JD, Winkler EA, Walker CT, Sullivan JS, Johnson M, Zlokovic BV. Deficiency in mural vascular cells coincides with blood-brain barrier disruption in Alzheimer's disease. Brain Pathol 2013;23:303-10.

37. Shankar GM, Walsh DM. Alzheimer's disease: synaptic dysfunction and Abeta. Mol Neurodegener 2009;4:48.

38. Selkoe DJ. Alzheimer's disease is a synaptic failure. Science 2002;298:789-91.

39. Lleo A, Nunez-Llaves R, Alcolea D, Chiva C, Balateu-Panos D, Colom-Cadena $\mathrm{M}$, et al. Changes in synaptic proteins precede neurodegeneration markers in preclinical Alzheimer's disease cerebrospinal fluid. Mol Cell Proteomics 2019;18:546-60.

40. Mirab F, Kang YJ, Majd S. Preparation and characterization of size-controlled glioma spheroids using agarose hydrogel microwells. PLoS One 2019;14:e0211078.

41. Serrati S, Martinelli C, Palazzo A, Iacobazzi RM, Perrone M, Ong QK, et al. Reproducibility warning: the curious case of polyethylene glycol 6000 and spheroid cell culture. PLoS One 2020;15:e0224002.

42. Li Y, Wang Y, Shen C, Meng Q. Non-swellable F127-DA hydrogel with concave microwells for formation of uniformsized vascular spheroids. RSC Adv 2020;10:44494-502.

43. Tung YC, Hsiao AY, Allen SG, Torisawa YS, Ho M, Takayama S. High-throughput 3D spheroid culture and drug testing using a 
384 hanging drop array. Analyst 2011;136:473-8.

44. Zanoni M, Piccinini F, Arienti C, Zamagni A, Santi S, Polico $\mathrm{R}$, et al. 3D tumor spheroid models for in vitro therapeutic screening: a systematic approach to enhance the biological relevance of data obtained. Sci Rep 2016;6:19103.

45. Nashimoto Y, Hayashi T, Kunita I, Nakamasu A, Torisawa YS, Nakayama M, et al. Integrating perfusable vascular networks with a three-dimensional tissue in a microfluidic device. Integr Biol (Camb) 2017;9:506-18.

46. Murphy SV, Atala A. 3D bioprinting of tissues and organs. Nat Biotechnol 2014;32:773-85.

47. Lorber B, Hsiao WK, Hutchings IM, Martin KR. Adult rat retinal ganglion cells and glia can be printed by piezoelectric inkjet printing. Biofabrication 2014;6:015001.

48. Xu T, Gregory CA, Molnar P, Cui X, Jalota S, Bhaduri SB, et al. Viability and electrophysiology of neural cell structures generated by the inkjet printing method. Biomaterials 2006; 27:3580-8.

49. Sharma R, Smits IPM, De La Vega L, Lee C, Willerth SM. 3D bioprinting pluripotent stem cell derived neural tissues using a novel fibrin bioink containing drug releasing microspheres. Front Bioeng Biotechnol 2020;8:57.

50. Gu Q Tomaskovic-Crook E, Lozano R, Chen Y, Kapsa RM, Zhou Q et al. Functional 3D neural mini-tissues from printed gel-based bioink and human neural stem cells. Adv Healthc Mater 2016;5:1429-38.

51. Miller JS, Stevens KR, Yang MT, Baker BM, Nguyen DH, Cohen DM, et al. Rapid casting of patterned vascular networks for perfusable engineered three-dimensional tissues. Nat Mater 2012;11:768-74.
52. Gao Q He Y, Fu JZ, Liu A, Ma L. Coaxial nozzle-assisted 3D bioprinting with built-in microchannels for nutrients delivery. Biomaterials 2015;61:203-15.

53. Gao G, Lee JH, Jang J, Lee DH, Kong JS, Kim BS, et al. Tissue engineered bio-blood-vessels constructed using a tissuespecific bioink and 3D coaxial cell printing technique: a novel therapy for ischemic disease. Adv Funct Mater 2017; 27:1700798.

54. Cho H, Hashimoto T, Wong E, Hori Y, Wood LB, Zhao L, et al. Microfluidic chemotaxis platform for differentiating the roles of soluble and bound amyloid- $\beta$ on microglial accumulation. Sci Rep 2013;3:1823.

55. Young EW, Beebe DJ. Fundamentals of microfluidic cell culture in controlled microenvironments. Chem Soc Rev 2010; 39:1036-48.

56. Halldorsson S, Lucumi E, Gomez-Sjoberg R, Fleming RM. Advantages and challenges of microfluidic cell culture in polydimethylsiloxane devices. Biosens Bioelectron 2015; 63:218-31.

57. Kim L, Toh YC, Voldman J, Yu H. A practical guide to microfluidic perfusion culture of adherent mammalian cells. Lab Chip 2007;7:681-94.

58. Raimondi I, Izzo L, Tunesi M, Comar M, Albani D, Giordano C. Organ-on-a-chip in vitro models of the brain and the bloodbrain barrier and their value to study the microbiota-gut-brain axis in neurodegeneration. Front Bioeng Biotechnol 2020; $7: 435$.

59. Zhang B, Korolj A, Lai BF, Radisic M. Advances in organ-on-achip engineering. Nat Rev Mater 2018;3:257-78. 